\title{
USP22 transcriptional activity is negatively regulated by the histone deacetylase inhibitor trichostatin $A$
}

\author{
JIANJUN XIONG ${ }^{1,2}$, XIAOYUAN XU ${ }^{1,2}$, XIAOU ZHOU ${ }^{1,2}$, JIANYUN LIU ${ }^{1}$, \\ ZHEN GONG ${ }^{2}$, PING WU ${ }^{1}$ and WEIDONG $\mathrm{LI}^{1}$ \\ ${ }^{1}$ Jiangxi Province Key Laboratory of Systems Biomedicine; \\ ${ }^{2}$ College of Basic Medical Science, Jiujiang University, Jiujiang, Jiangxi 332000, P.R. China
}

Received November 29, 2013; Accepted August 29, 2014

DOI: $10.3892 / \mathrm{mmr} .2014 .2666$

\begin{abstract}
The ubiquitin-specific protease 22 (USP22) gene is overexpressed in the majority of types of cancer cells, and has been implicated in tumorigenesis. However, the mechanisms that regulate its expression remain unclear. The results of the present study demonstrated that the expression of USP22 is negatively regulated by trichostatin A (TSA), a classical histone deacetylase inhibitor. Furthermore, TSA was revealed to interfere with the binding of RNA polymerase II to the USP22 promoter, directly suppressing its transcription. In addition, the overexpression of USP22 was observed to attenuate TSA-induced apoptosis in HeLa cells. To the best of our knowledge, these results provide the first insight into the regulation of the USP22 gene by antitumor drugs and into the mechanisms underlying the anticancer activity of TSA.
\end{abstract}

\section{Introduction}

The human ubiquitin-specific protease 22 (USP22) gene is located on chromosome 17p11.2. Its product is a 525 amino acid protein with a molecular weight of $\sim 60 \mathrm{kDa}$ that functions as a deubiquitinating enzyme in vivo and in vitro (1). In humans, USP22 is a subunit of the SAGA coactivator complex that is required for activator-driven transcription. Substrates of USP22 include the histones $\mathrm{H} 2 \mathrm{~A}$ and $\mathrm{H} 2 \mathrm{~B}$, the shelterin protein TRF1, the transcription factor p53, and FBP1 (2-5). In addition, USP22 is overexpressed in numerous human tumors and is associated with treatment resistance, tumor aggressiveness and increased metastasis in cancer patients $(6,7)$. However, little is known regarding the transcriptional regulation of USP22.

Correspondence to: Professor Weidong Li, Jiangxi Province Key Laboratory of Systems Biomedicine, Jiujiang University, 17 Lufeng Road, Jiujiang, Jiangxi 332000, P.R. China

E-mail: 1wd626518@163.com

Key words: ubiquitin-specific protease 22, promoter, trichostatin A, luciferase
Acetylation is a posttranslational modification that regulates the physiological functions of proteins. Histone acetylases (HAT) and deacetylases (HDACs) regulate the dynamic equilibrium of acetylation (8). HDAC inhibitors have been identified as a novel class of potential drugs for use in cancer therapy, based on observations that the overexpression of HDAC was associated with tumorigenesis (9). The antitumor effects of HDAC inhibitors are due to the transcriptional alteration of specific cancer-related genes, including regulators of the cell cycle, apoptosis, differentiation and invasion (10-12). Therefore, it is hypothesized that HDAC-induced apoptosis may alter USP22 expression.

The aim of the present study was to determine the effect of the HDAC inhibitor trichostatin A (TSA) on the expression levels of endogenous USP22 and its promoter activity in human cancer cells. In addition, the mechanisms underlying the anticancer activity of TSA were investigated.

\section{Materials and methods}

Cell culture. Human HeLa cells (American Type Culture Collection, Manassas, VA, USA) were used in this study. The cells were cultured in Dulbecco's modified Eagle's medium (DMEM, Invitrogen Life Technologies, Carlsbad, CA, USA) supplemented with $10 \%$ fetal bovine serum (Gibco-BRL, Carlsbad, CA, USA) at $37^{\circ} \mathrm{C}$ with $5 \% \mathrm{CO}_{2}$.

Total RNA isolation and reverse transcription-quantitative polymerase chain reaction ( $R T-q P C R)$. Total RNA extraction and qPCR were performed as described previously (13). Briefly, total RNA was isolated from cells using TRIzol Reagent (Invitrogen), and reverse-transcribed using M-MLV reverse transcriptase. Real-time PCR was performed using SYBR Green PCR Master Mix (Takara Bio, Dalian, China) on an ABI 7500 Real-Time PCR System (Applied Biosystems, Carlsbad, CA, USA). The mRNA levels of USP22 and GAPDH were measured with the following specific primers: Forward: 5'-GTGTCTTCTTCGGCTGTTTA-3' and reverse: 5'-CTC CTCCTTGGCGATTATTT-3' for USP22; and forward: 5'-AGAAGGCTGGGGCTCATTTG-3' and reverse: 5'-AGG GGCCATCCACAGTCTTC-3' for GAPDH. All qPCR results are presented as the mean of at least three independent experiments using duplicate qPCR analysis. 
Western blotting. Whole cell lysates were separated by SDS-PAGE using $10 \%$ polyacrylamide gels and transferred to polyvinylidene fluoride membranes (Bio-Rad, Hercules, CA, USA). After overnight blocking at $4^{\circ} \mathrm{C}$ in a buffer containing $5 \%(\mathrm{wt} / \mathrm{vol})$ non-fat milk powder, the membrane was incubated in fresh buffer with the appropriate antibody for $1 \mathrm{~h}$ at room temperature. Monoclonal antibodies against USP22 and GAPDH were purchased from Santa Cruz Biotechnology, Inc. (sc-69082 and sc-20358; Dallas, TX, USA). The antigenantibody complex was detected by incubating the membrane for $1 \mathrm{~h}$ at room temperature in buffer containing a 1:10,000 dilution of horseradish peroxidase-conjugated anti-goat IgG secondary antibody (Santa Cruz Biotechnology) Western blots were visualized using an enhanced chemiluminescence system (Pierce Biotechnology, Inc. Rockford, IL, USA).

Plasmid constructs. The USP22 promoters were constructed as described previously (13). To construct CMV-driven USP22 expression vectors, total RNA was isolated from human HeLa cells and reverse-transcribed using the reverse transcriptase M-MLV primed by an oligo (dT)15 primer. The primers used in the subsequent PCR amplification of USP22 cDNA were as follows: Forward: 5'-GAAGCTTATGAGCGACCAAGA TCACTCCATG-3' and reverse: 5'-GGAATTCTCAGAAGC CATTGCCACTGA-3'. The PCR products were analyzed on agarose gels and digested with HindIII and EcoRI and ligated into pCMV-His with T4 DNA ligase (Takara Bio). The fidelity of the constructs was confirmed by sequencing. The sequencing primer was TAATACGACTCACTATAGGG.

Luciferase assay. Cells were plated onto 24-well plates at a density of $5 \times 10^{4}$ cells per well and left overnight. This was followed by transfection with promoter reporter plasmid DNA using Lipofectamine 2000 reagent (Invitrogen Life Technologies). The media were changed $24 \mathrm{~h}$ later, for medium with or without TSA, and cell lysates were collected at the times indicated for analysis by luciferase assay using a Dual Luciferase Assay kit (Promega, Madison, WI, USA). All transfections were performed in triplicate.

Chromatin immunoprecipitation (ChIP). ChIP was performed as described previously (13). Briefly, cells were fixed by adding formaldehyde to a final concentration of $1 \%$ and incubated with moderate shaking for $30 \mathrm{~min}$ at room temperature. Thereafter, cells were washed twice with cold phosphate-buffered saline (PBS). The pellet was resuspended and lysed, and nuclei were isolated and sonicated until the chromatin had an average length of 500-1500 bp. Chromatin fragments were immunoprecipitated from cells using rabbit polyclonal antibody against RNA polymerase II (sc-899x; Santa Cruz Biotechnology) DNA samples from the immunoprecipitates and control inputs were analyzed using QPCR with the following primers: forward, 5'-GTCTACCCAGAGCCT AACGG-3', and reverse 5'-GCGGAGGCCGGACAAAGA TGGG-3'.

Flow cytometry. Cells $\left(1 \times 10^{5}\right)$ were seeded onto 6-well plates, incubated overnight and transfected with USP22 or an empty vector. The media were changed $24 \mathrm{~h}$ later for media containing TSA, and cells were incubated for an additional 24 or $48 \mathrm{~h}$. The cells were then harvested using trypsin, washed with $1 \mathrm{X}$ PBS, and resuspended in $50 \mu \mathrm{l}$ PBS. Cell death was assessed by flow cytometry (FACSCalibur; BD Biosciences, Franklin Lakes, NJ, USA) following staining with fluorescein isothiocyanate-Annexin $\mathrm{V}$ and potassium iodide.

Statistical analysis. Each experiment was performed at least three times independently. The SPSS 13.0 software program (SPSS, Inc., Chicago, IL, USA) was used for statistical analysis. The data between two groups was analyzed by a Student's $\mathrm{t}$-test. The data between multiple groups were analyzed by one-way analysis of variance. $\mathrm{P}<0.05$ was considered to indicate a statistically significant difference.

\section{Results}

TSA suppresses USP22 expression. To assess the effects of HDAC inhibitors on USP22 expression, HeLa cells were incubated with apoptosis-inducing concentrations of TSA (330 nM) (14) for different time periods, and USP22 mRNA levels were examined by RT-qPCR. As shown in Fig. 1A, the USP22 mRNA expression levels were reduced marginally following a $3 \mathrm{~h}$ incubation with TSA and were reduced significantly after 6-12 h, indicating that the suppression is a time-dependent. The effects of TSA on the expression levels of the USP22 protein were determined by western blotting. Consistent with the reduction in USP22 mRNA, TSA downregulated the expression levels of USP22 protein (Fig. 1B), with a significant reduction following $24 \mathrm{~h}$ of treatment. In addition, TSA downregulated the expression of USP22 in another cancer cell line, HepG2 (data not shown).

To exclude the possibility that TSA affected the stability of USP 22 mRNA, actinomycin D was used to block mRNA transcription, and the levels of USP22 mRNA were assessed with RT-qPCR. When cells were maintained in normal culture conditions, the half-life of USP $22 \mathrm{mRNA}$ was $\sim 6 \mathrm{~h}$ following the inhibition of transcription. When cells were cotreated with TSA and actinomycin D the half-life of USP 22 mRNA was unchanged, indicating that TSA does not affect the stability of USP22 mRNA.

TSA suppresses USP22 promoter activity. Subsequently, luciferase assays were used to assess the effects of TSA on the activity of the USP 22 promoter. The $2.8 \mathrm{~kb}$ wild-type USP22 promoter P-2828/+52 was transfected into HeLa cells and incubated for $24 \mathrm{~h}$. Following incubation, cells were treated with TSA for different time periods. As shown in Fig. 2A, treatment with TSA reduced the promoter activity in a time-dependent manner, which is consistent with the reduction of the USP22 mRNA expression levels. Subsequently, a series of truncated promoter-reporter gene constructs, derived from p-2828/+52, were transfected into cells to characterize the regulatory sequence that responds to TSA. Notably, the promoter activity of the $5^{\prime}$ deletion constructs p-2828/+52, p-1306/+52, p-210/+52, and p-7/+52 and the 3 deletion constructs p-210/+31, p-210/+11 were all reduced by treatment with TSA compared with that of the untreated cells (Fig. 2B). Finally, the activity of a promoter harboring a mutated Sp1 binding site ( $\mathrm{p}-210 / \mathrm{sp} 1 \mathrm{mut})$ located between -7 and -13 was also reduced by $40 \%$ following treatment with TSA compared with that of the wild-type promoter p-210/+52 (Fig. 2C). This suggests that the Sp1 binding 
A

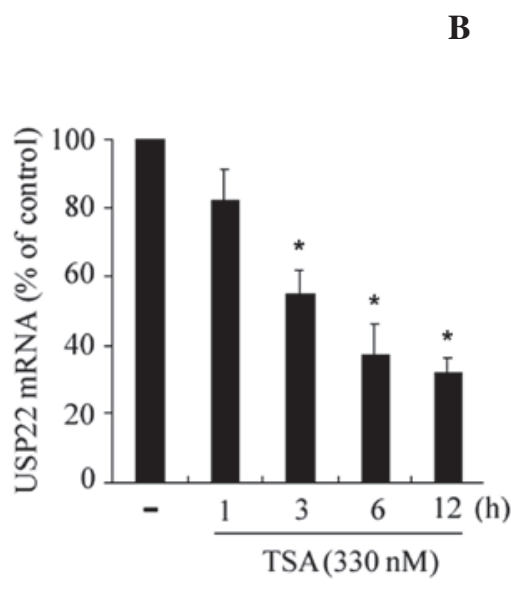

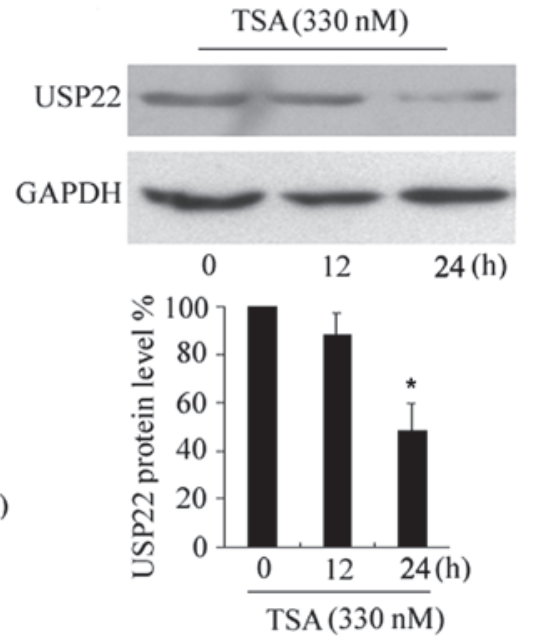

Figure 1. (A) Reverse transcription-quantitative polymerase chain reaction (RT-qPCR) analysis of USP22 mRNA expression in HeLa cells. Cells were treated with $330 \mathrm{nM}$ trichostatin A (TSA) for 3, 6 or $12 \mathrm{~h}$. Cells were harvested and their RNA was isolated, reverse transcribed into cDNA, and the expression levels of USP22 mRNA were determined by RT-qPCR. Values are presented as the mean \pm standard error of the mean of three independent experiments. (B) Western blot analysis of USP22 protein levels following treatment with $330 \mathrm{nM}$ TSA for 12 or $24 \mathrm{~h}$. TSA significantly decreased USP22 protein level after $24 \mathrm{~h}$ incubation. ${ }^{*} \mathrm{P}<0.05$, one-way analysis of variance.

A

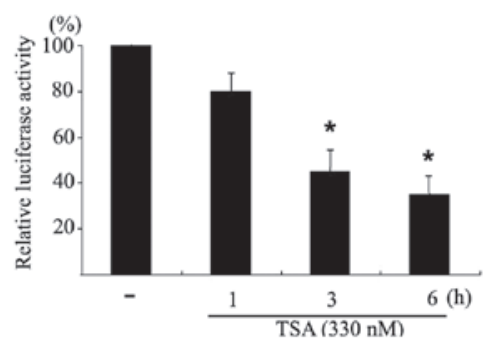

B

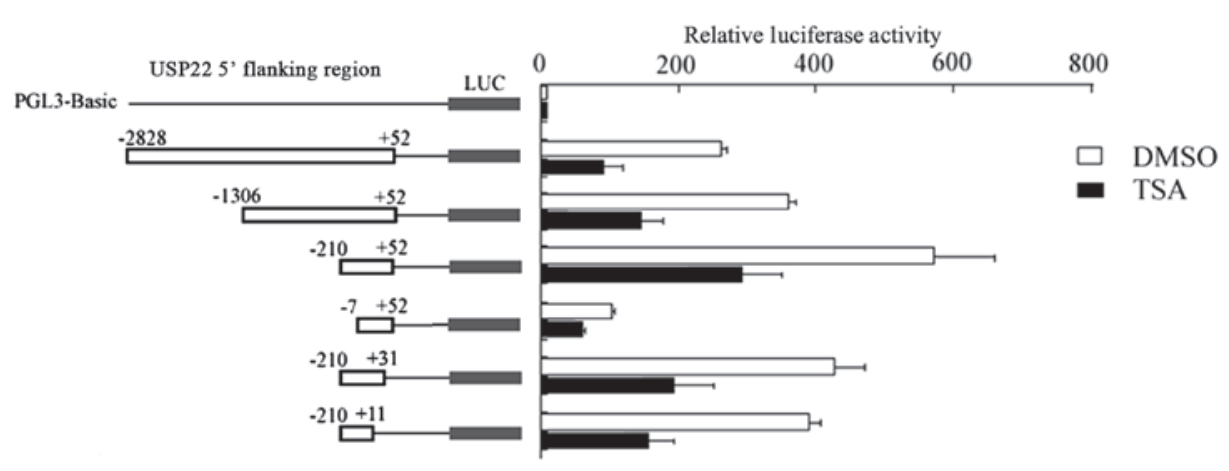

$\mathbf{C}$

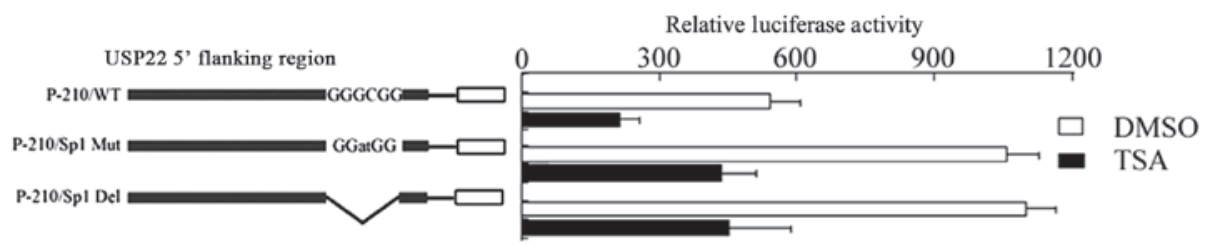

Figure 2. Effects of trichostatin A (TSA) on USP22 promoter activity. (A) HeLa cells were transfected with the $2.8 \mathrm{~kb}$ USP22 promoter, treated with $330 \mathrm{nM}$ TSA for 1,3 or $6 \mathrm{~h}$ and the luciferase activity was measured TSA decreased USP22 promoter activity in a time-dependent manner. (B) HeLa cells were transfected with different constructs of USP22 promoters, treated with $330 \mathrm{nM}$ TSA for $6 \mathrm{~h}$ and the luciferase activity was measured. TSA decreased USP22 promoter activity in a time-dependent manner. (C) HeLa cells were transfected with p-210/splmut, treated with $330 \mathrm{nM}$ TSA for $6 \mathrm{~h}$ and the luciferase activity was measured. Sp1 binding site is not involved in the TSA-mediated suppression of USP22 promoter activity. ${ }^{*} \mathrm{P}<0.05$, one-way analysis of variance.

site is not involved in the TSA-mediated suppression of USP22 promoter activity.

TSA-induced downregulation of USP22 involves the recruitment of RNA polymerase II. Recent studies have demonstrated that HDAC activity is required for the recruitment of RNA polymerase II to several specific gene promoters (15). Since no regulatory elements were characterized that were responsive to TSA repression up or downstream of the USP22 gene transcription start site, the effect of TSA on the recruitment 


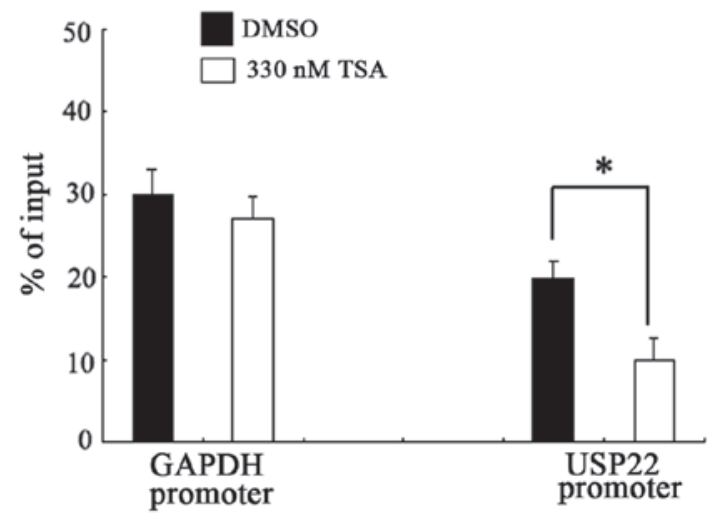

Figure 3. Trichostatin A (TSA) inhibits the recruitment of RNA polymerase II to the USP22 promoter in HeLa cells. Cells were pretreated with $330 \mathrm{nM}$ TSA for $6 \mathrm{~h}$. Cross-linked DNA-protein complexes were then immunoprecipitated using anti-RNA polymerase II antibodies, and amplified by polymerase chain reaction using specific primers designed for the USP22 promoter and GAPDH promoter regions, containing the transcription start site. The data are representative of three independent experiments. ${ }^{*} \mathrm{P}<0.05$.

A
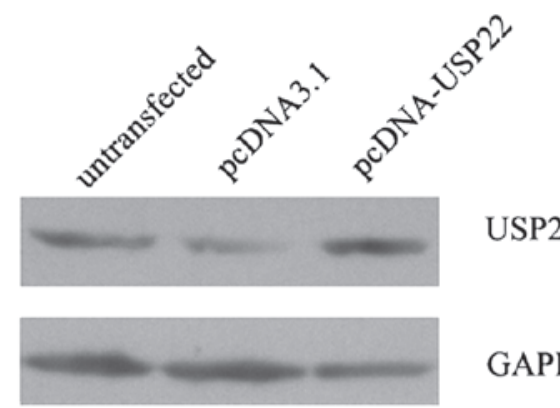

\section{GAPDH}

B
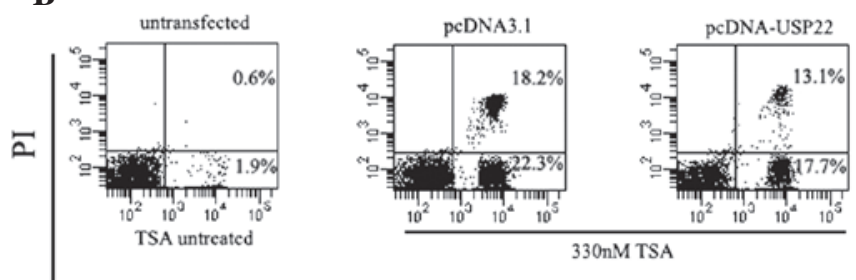

AnnexinV-FITC

Figure 4. Untransfected HeLa cells and cells transiently transfected with an empty vector or a USP22 expression plasmid were treated with $330 \mathrm{nM}$ trichostatin A (TSA) for $24 \mathrm{~h}$. Cells were then stained using propidium iodide and fluorescein isothiocyanate-Annexin $\mathrm{V}$, and analyzed by flow cytometry using a BD FACScan flow cytometer.

of RNA polymerase II to the USP22 promoter was investigated using a ChIP assay. As shown in Fig. 3, TSA treatment significantly blocked the binding of RNA polymerase II to the USP22 promoter in HeLa cells. This inhibitory effect was not detected with the control promoter region of GAPDH, indicating that the inhibition was specific to the USP 22 promoter.

Overexpression of USP22 attenuates TSA-induced apoptosis. The effect of USP22 overexpression on TSA-induced apoptosis was investigated. A CMV-driven USP22 expression vector was generated and used to transiently transfect HeLa cells. As shown in Fig. 4, a significant increase in the expres- sion levels of USP22 protein was observed in pcDNA-USP22 transfected cells, whereas no change was detected in empty vector-transfected cells. As expected, the overexpression of USP22 significantly decreased TSA-induced apoptosis in HeLa cells (Fig. 4).

\section{Discussion}

Recently, research interest in the expression of USP 22 has increased. In embryogenesis, USP22 is expressed periodically and its depletion may lead to early embryonic mortality. Furthermore, the overexpression of USP22 has been observed in the majority of types of human cancer cell and has been linked to cancer progression $(2,6)$. Therefore, the reduction of USP22 expression levels may provide a novel cancer therapy. However, the mechanisms that regulate USP22 expression, particularly in human tumor cells remain to be elucidated. The results of the current study revealed for the first time that a HDAC inhibitor negatively regulates USP22 expression.

TSA is a classic HDAC inhibitor that induces apoptosis by altering the expression levels of pro- and anti-apoptotic genes. For example, the expression levels of the cyclin-dependent kinase inhibitor p21 were upregulated during TSA-induced apoptosis (12), whereas the expression levels of survivin, an apoptosis inhibitor, were reduced (11). As USP22 is an anti-apoptotic protein, it was hypothesized that USP22 is downregulated by TSA, a hypothesis that was supported by the results of the GPCR and western blot analysis. However, the mechanisms that regulate TSA-induced gene silencing are complex, and not completely understood. Certain studies have indicated that TSA reduces gene expression at the transcriptional level (10), whereas others reported that TSA suppresses gene expression by affecting mRNA stability (16). An additional study revealed that TSA may lead to protein degradation (17). In the present study, TSA inhibited USP22 expression at the transcription level, and did not affect the half-life of USP 22 mRNA. The TSA-mediated suppression of USP 22 was an early, time-dependent event. These results are supported by a previous study that determined that USP 22 transcription is regulated by extracellular signals (18).

Since TSA suppressed the expression levels of USP 22 mRNA, one of the aims of the current study was to characterize the specific elements in the USP22 promoter that were targeted by TSA. Although several regulatory elements were found in the USP22 promoter, no DNA element up- or downstream of the transcription start site was responsive to TSA, including the Sp1 binding site that was identified close to the USP22 transcription start site. A previous study suggested that $\mathrm{Sp} 1$ is important in HDAC inhibitor-induced apoptosis (19). Sp1 is a transcription factor that is acetylated in the DNA binding domain by $\mathrm{CBP} / \mathrm{p} 300$ and subsequently modulates transcriptional activity and influences protein-protein interactions (20). Therefore, it was originally hypothesized that TSA-stimulated suppression of USP22 expression was mediated by this site. However, promoter deletion and mutagenesis analyses showed that the Sp1 binding site was not involved in TSA-induced USP22 downregulation.

TSA responsive regions were not identified up- or downstream of the USP22 transcription start site, indicating that the key region that mediates TSA-induced USP22 suppres- 
sion may be located close to the transcription initiation site. A previous study suggested that histone deacetylase activity is required to recruit RNA polymerase II to promoters, thus affecting gene transcription (15). Therefore, interfering with RNA polymerase recruitment is one mechanism by which HDAC inhibitors stimulate gene silencing (21). In the present study, ChIP analysis revealed that TSA blocked the recruitment of RNA polymerase II, indicating that TSA blocks the formation of the preinitiation complex at the USP22 promoter. Nevertheless, the mechanism by which HDAC activity affected the binding of RNA polymerase II to the USP22 promoter requires further investigation.

To confirm the anti-apoptotic role of TSA treatment, a USP22 expression vector driven by a CMV promoter was produced. Since the activity of the CMV promoter increases marginally in response to TSA treatment (22), it was possible to obtain USP22 over-expressing HeLa cells following treatment with TSA. The overexpression of USP22 attenuates TSA-induced apoptosis, indicating that USP22 has an important role in HDAC inhibitor-induced apoptotic signaling. Although the mechanism by which USP22 attenuates TSA-induced apoptosis is not yet fully understood, the regulation of apoptosis-related genes by USP22 is a possible route. Indeed, several key genes in TSA-induced apoptosis, such as the cyclin-dependent kinase inhibitor p21 WAF1, are also modulated by USP22. $p 21$ is a downstream target gene of USP22, and downregulation of USP22 promoted p21 expression. By contrast, p21 is upregulated during TSA-induced apoptosis. Therefore, the suppression of USP22 expression by TSA may regulate p21 expression. In addition, p53 may also participate in the TSA-USP22 pathway as it has been linked to TSA-induced apoptosis. Whilst USP22-mediated stabilization of SIRT1 has been shown to lead to a reduction in the levels of p53 acetylation and the suppression of p53-mediated apoptosis (4), contradictory studies have reported that the activity and stability of SIRT1 are not affected by USP22 (23). These contrasting results could be due to cell-specific effects. A greater understanding of the mechanisms by which USP22 regulates gene transcription may identify additional molecules that are involved in TSA-induced apoptosis.

In conclusion, the present study revealed that the expression of USP22 is negatively regulated by TSA. Furthermore, it was determined that this negative regulation occurred via interference of the binding between RNA polymerase II and the USP22 promoter. In addition, the exogenous overexpression of USP22 attenuated TSA-induced apoptosis. These results indicate that blocking USP22 expression at the transcriptional level by TSA may provide a novel strategy for cancer therapy.

\section{Acknowledgements}

This study was supported by the National Nature Science Foundation of China (grant no. 31000581) and the Jiangxi Science and Technology Support Programmer, P.R. China (grant no. 2010BSA14000).

\section{References}

1. Lee HJ, Kim MS, Shin JM, Park TJ, Chung HM and Baek KH: The expression patterns of deubiquitinating enzymes, USP22 and Usp22. Gene Expr Patterns 6: 277-284, 2006.
2. Zhang XY, Varthi M, Sykes SM, et al: The putative cancer stem cell marker USP22 is a subunit of the human SAGA complex required for activated transcription and cell-cycle progression. Mol Cell 29: 102-111, 2008

3. Zhao Y, Lang G, Ito S, et al: A TFTC/STAGA module mediates histone $\mathrm{H} 2 \mathrm{~A}$ and $\mathrm{H} 2 \mathrm{~B}$ deubiquitination, coactivates nuclear receptors, and counteracts heterochromatin silencing. Mol Cell 29: 92-101, 2008.

4. Lin Z, Yang H, Kong Q, et al: USP22 antagonizes p53 transcriptional activation by deubiquitinating Sirtl to suppress cell apoptosis and is required for mouse embryonic development Mol Cell 46: 484-494, 2012.

5. Atanassov BS and Dent SY: USP22 regulates cell proliferation by deubiquitinating the transcriptional regulator FBP1. EMBO Rep 12: 924-930, 2011.

6. Glinsky GV: Death-from-cancer signatures and stem cell contribution to metastatic cancer. Cell Cycle 4: 1171-1175, 2005.

7. Glinsky GV, Berezovska O and Glinskii AB: Microarray analysis identifies a death-from-cancer signature predicting therapy failure in patients with multiple types of cancer. J Clin Invest 115: 1503-1521, 2005.

8. Kouzarides T: Acetylation: a regulatory modification to rival phosphorylation? EMBO J 19: 1176-1179, 2000.

9. Kawai H, Li H, Avraham S, Jiang S and Avraham HK: Overexpression of histone deacetylase HDAC1 modulates breast cancer progression by negative regulation of estrogen receptor alpha. Int J Cancer 107: 353-358, 2003.

10. Duan H, Heckman CA and Boxer LM: Histone deacetylase inhibitors down-regulate bcl-2 expression and induce apoptosis in $\mathrm{t}(14 ; 18)$ lymphomas. Mol Cell Biol 25: 1608-1619, 2005.

11. Hsu YF, Sheu JR, Lin CH, et al: Trichostatin A and sirtinol suppressed survivin expression through AMPK and p38MAPK in HT29 colon cancer cells. Biochim Biophys Acta 1820: 104-115, 2012.

12. Han JW, Ahn SH, Kim YK, et al: Activation of p21(WAF1/Cip1) transcription through $\mathrm{Sp} 1$ sites by histone deacetylase inhibitor apicidin: involvement of protein kinase C. J Biol Chem 276: 42084-42090, 2001

13. Xiong J, Che X, Li X, Yu H, Gong Z and Li W: Cloning and characterization of the human USP22 gene promoter. PLoS One 7: e52716, 2012

14. Sakamoto S, Potla R and Larner AC: Histone deacetylase activity is required to recruit RNA polymerase II to the promoters of selected interferon-stimulated early response genes. J Biol Chem 279: 40362-40367, 2004.

15. Zhang Y and Dufau ML: Silencing of transcription of the human luteinizing hormone receptor gene by histone deacetylase-mSin3A complex. J Biol Chem 277: 33431-33438, 2002.

16. Xiong Y, Dowdy SC, Podratz KC, et al: Histone deacetylase inhibitors decrease DNA methyltransferase-3B messenger RNA stability and down-regulate de novo DNA methyltransferase activity in human endometrial cells. Cancer Res 65: 2684-2689, 2005.

17. Chen WY, Weng JH, Huang CC and Chung BC: Histone deacetylase inhibitors reduce steroidogenesis through SCF-mediated ubiquitination and degradation of steroidogenic factor 1 (NR5A1). Mol Cell Biol 27: 7284-7290, 2007.

18. Ovaa H, Kessler BM, Rolen U, Galardy PJ, Ploegh HL and Masucci MG: Activity-based ubiquitin-specific protease (USP) profiling of virus-infected and malignant human cells. Proc Natl Acad Sci USA 101: 2253-2258, 2004.

19. Waby JS, Chirakkal H, Yu C, et al: Sp1 acetylation is associated with loss of DNA binding at promoters associated with cell cycle arrest and cell death in a colon cell line. Mol Cancer 9: 275, 2010.

20. Suzuki T, Kimura A, Nagai R and Horikoshi M: Regulation of interaction of the acetyltransferase region of p300 and the DNA-binding domain of Sp1 on and through DNA binding. Genes Cells 5: 29-41, 2000.

21. Furumai R, Ito A, Ogawa K, et al: Histone deacetylase inhibitors block nuclear factor-kappaB-dependent transcription by interfering with RNA polymerase II recruitment. Cancer Sci 102: 1081-1087, 2011

22. Choi KH, Basma H, Singh J and Cheng PW: Activation of CMV promoter-controlled glycosyltransferase and beta-galactosidase glycogenes by butyrate, tricostatin A, and 5-aza-2'-deoxycytidine. Glycoconj J 22: 63-69, 2005.

23. Armour SM, Bennett EJ, Braun CR, et al: A high-confidence interaction map identifies SIRT1 as a mediator of acetylation of USP22 and the SAGA coactivator complex. Mol Cell Biol 33: 1487-1502, 2013. 